'Escuela de Enfermería, Pontificia Universidad Católica de Chile. Santiago, Chile. ${ }^{2}$ Facultad de Enfermería, Universidad Andres Bello. Santiago, Chile. aEnfermera, Estudiante de Magíster en Enfermería.

bPhD, EM, Profesor Investigador.

*Este artículo es producto de un estudio financiado por la Comisión Nacional de Investigación Científica y Tecnológica del Gobierno de Chile-CONICYT, a través de proyecto FONDECYT

№ 11130568. La organización que la proporcionó el financiamiento no tuvo influencia en el diseño del estudio: en la recolección, análisis o interpretación de los datos; en la preparación, revisión o aprobación del manuscrito.

Recibido el 24 de diciembre de 2015, aceptado el 29 abril de 2016.

Correspondencia a: Alejandra-Ximena Araya. Sazié 2212, Santiago, Chile. Teléfono: (56) 226618903 alejandra.araya.g@unab.cl

\section{Criterios de fragilidad en personas mayores que viven en la comunidad: una actualización de la literatura}

\author{
EVELYN IRIARTE ${ }^{1, a}$, ALEJANDRA-XIMENA ARAYA ${ }^{2, b}$
}

\section{Frailty criteria among older people living in the community. An update}

Frailty is an important concept in aging research and care. Awareness of frailty signs allows healthcare workers to identify older adults at risk and design interventions to prevent their functional decline, therefore preventing adverse health outcomes. This literature review allows to identify predictors of frailty considering its physical, psychological and social domains. From the evidence found, it is possible to formulate a profile of fragile older people. Finally, future research should focus on the description of psychological and social fragility profiles, on the detection of persons likely to be fragile and finally to define precise instruments to detect fragile people, which should become the gold standards in future research.

(Rev Med Chile 2016; 144: 1440-1447)

Key words: Aged; Frail Elderly; Health Services for the Aged; Risk Management.

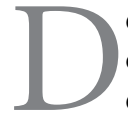
esde 1978, cuando el "Federal Council on Aging" en Estados Unidos introdujo el término "anciano frágil", la fragilidad se ha convertido en un importante concepto en la investigación sobre el envejecimiento y el cuidado de personas mayores ${ }^{1}$.

Gobbens, Luijkx, Wijnen-Sponselee \& Schols ${ }^{2}$ definen la fragilidad como "un estado dinámico que afecta a un individuo que experimenta pérdidas en uno o más dominios del funcionamiento humano (físico, psicológico y social) que son causadas por la influencia de una gama de variables, y que aumenta el riesgo de resultados adversos". Dicho término contiene un enfoque multidimensional; es dinámico; tiene validez predictiva para los resultados adversos; no incluye la enfermedad, la comorbilidad, o discapacidad; y es medible en la práctica clínica.

La prevalencia de fragilidad es variada a nivel mundial, fluctúa entre 14-43\% dependiendo de la definición e instrumento de medición empleado ${ }^{3-5}$. El conocimiento del concepto de fragilidad, desde una perspectiva amplia, permite que los trabajadores de la salud que están en contacto con personas mayores puedan identificarlos $y$, consecuentemente, establecer intervenciones para evitar el exceso de declinamiento funcional de estas, evitando resultados adversos en salud ${ }^{1}$. El objetivo de esta revisión es actualizar la definición de fragilidad desde una perspectiva física, psicológica y social según el modelo de Gobbens y otros.

\section{Material y Métodos}

Para la búsqueda de los artículos de investigación fueron seleccionadas las siguientes bases de datos: The Cumulative Index to Nursing and Allied Health Literature (CINAHL), The American Psychological Association's PsycINFO ${ }^{\circledR}$, PubMed and Scientific Electronic Library Online SciELO. Estas bases de datos fueron escogidas ya que cuentan con los recursos más completos en salud, psicología y literatura sudamericana. 
Los criterios de inclusión para la selección de los artículos fueron:

- Estudios observacionales, de intervención y cualitativos que describan los predictores de fragilidad en personas mayores que viven en la comunidad.

- Artículos publicados en idioma inglés, español o portugués publicados en los últimos 10 años (enero de 2001 hasta octubre de 2012).

- Artículos que consideren en la muestra sólo participantes personas mayores de 60 años.

Los criterios de exclusión para la selección de los artículos fueron:

- La atención pediátrica o maternal.

- Investigaciones realizadas en el servicio de hospitalizados, urgencia, establecimientos de larga estadía, hospital de día y centros de día.

- El acceso sólo a resúmenes, revisiones bibliográficas y tesis de pre y postgrado.

La búsqueda en las bases de datos utilizó los siguientes descriptors: Frail Elderly"[Majr] OR frail $^{\star}[\mathrm{TI}]$ ) AND (Predict*[TIAB]) AND ("Aged"[Majr] OR aged [TI] OR elderly [TI] OR "old adult" [TI] OR "older people" [TI] OR "older person" [TI] OR aging [TI] OR geriatric $[\mathrm{TI}]$ ) AND communit ${ }^{\star}$ [all fields]. Se ocuparon los recursos de información bibliográfica de la Pontificia Universidad Católica de Chile para la extracción de los artículos de investigación según protocolo de selección.

En total se analizaron 38 artículos (Figura 1). Las secciones de los artículos de investigación utilizada para el análisis fueron: Métodos, Resultados y Conclusiones. Siguiendo los principios de análisis de contenido, se incluyó la identificación de los significados que se mencionaban de forma más repetitiva, la consistencia de éstos, las explicaciones y relaciones de éstos a través de todos los estudios incluidos en esta revisión. De esta forma los resultados fueron organizados, según el modelo de Gobbens ${ }^{1}$.

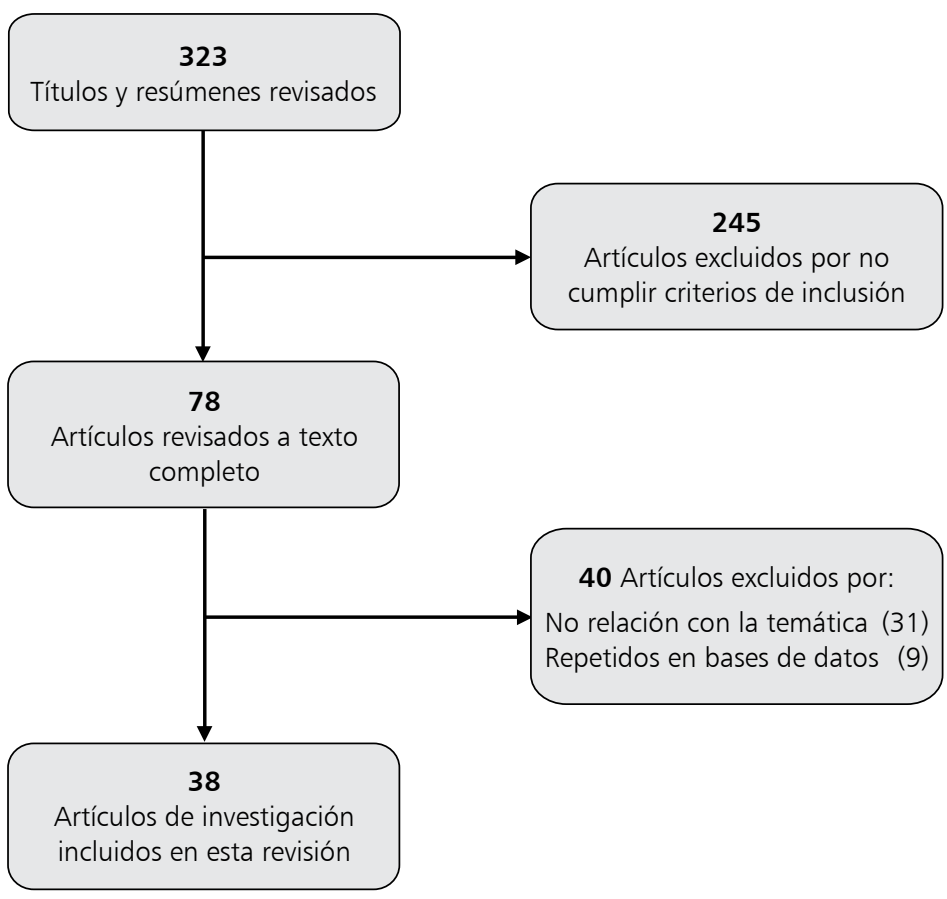

Figura 1. Flujograma de inclusión y exclusión de artículos. 


\section{Resultados}

Posterior al análisis de los artículos seleccionados, los resultados fueron agrupados de la siguiente manera (ver Tabla 1):

\section{Determinantes del curso de la vida}

Biología y genética. Según un estudio realizado en Estados Unidos en el año 2006, concluyó que el riesgo de fragilidad aumenta con el número deficiente de micronutrientes ${ }^{6}$. Asimismo, en un estudio realizado en 2009 en dicho país, dirigido a evaluar la expresión de CXCL-10 (quimiocina proinflamatoria) se encontró que los participantes frágiles tenían mayores niveles de expresión de esta que los no frágiles. Así, se concluyó que la expresión de esta es un mecanismo molecular importante que contribuye a la activación inflamatoria en los adultos mayores frágiles ${ }^{7}$.

Sumado a lo anterior, existe evidencia que data desde el año 2010 proveniente de un estudio Australiano que estableció que bajos niveles de Alanina Transaminasa (ALT) se asoció con fragilidad. La baja actividad de esta es un predictor de la reducción de la supervivencia; sin embargo, esto parece estar mediado por su asociación con la fragilidad y la edad ${ }^{8}$.

En cuanto a andrógenos y estrógenos, de acuerdo a un estudio realizado en Australia en el año 2011, se mostraron asociaciones ajustadas por edad significativas con la fragilidad concurrente. Los sujetos en el quintil más bajo de estas sustancias tenían probabilidades 2,2 veces de exhibir una mayor fragilidad física en comparación con el quintil más alto. Se concluyó que cambios relacionados con la edad en los andrógenos y estrógenos en la sangre pueden contribuir al desarrollo o la progresión de la fragilidad en hombres ${ }^{9}$.

Existe evidencia proveniente de un estudio realizado en el año 2013 en Reino Unido, que concluyó que niveles basales más altos de recuentos de leucocitos y cortisol, así como niveles bajos de DHEAS, se asociaron con aumento de todas las probabilidades de fragilidad en 10 años de seguimiento. Asimismo, se concluyó que biomarcadores de sustancias inmune-endocrinas están asociados con probabilidad de fragilidad, así como la mortalidad durante un período de 10 años ${ }^{10}$. Sumado a lo previo, un estudio realizado en Estados Unidos en el año 2009, encontró una asociación entre fragilidad y los niveles de DHEAS ${ }^{11}$.
Siguiendo la línea de lo anterior, los recuentos altos de neutrófilos y de monocitos se asociaron con fragilidad en mujeres de edad avanzada con discapacidad ${ }^{12}$. Se han evidenciado niveles más altos de IL-6 y Proteína C reactiva asociados a fragilidad ${ }^{13}$. Asimismo, niveles bajos de eGFR-cystatina estaban asociados con un mayor riesgo de fragilidad prevalente e incidente. Bajos niveles de eGFR serum creatinina no se asociaban a fragilidad ${ }^{14}$.

Edad y sexo. Según un estudio realizado en siete ciudades de Latinoamérica en el año 2008, se encontró que la niñez (hambre, mal estado salud, y condiciones socioeconómicas pobres) estaba asociado con fragilidad ${ }^{15}$. Asimismo, de acuerdo a Song en el año 2010, para los mayores de 85 años, el índice de fragilidad identificó 39,1\% de los hombres como frágil, en comparación con 45,1\% de las mujeres. En este estudio se concluyó que la prevalencia de fragilidad aumenta con la edad y en cualquier edad disminuye la supervivencia ${ }^{16}$. Asimismo, de acuerdo a un estudio realizado en el año 2010, se estableció que los efectos de otros determinantes difieren entre dominios de fragilidad; por ejemplo, la edad predice fragilidad física ${ }^{17}$. Lo anterior es concordante con un estudio realizado en Brasil que estableció que la edad cronológica se asociaba a fragilidad ${ }^{18}$. Sumado a lo anterior, en un estudio realizado en el año 2015 en México, se encontró que $37 \%$ de la muestra reunió los criterios de fragilidad. Éstos, en comparación con los clasificados como no frágiles, fueron significativamente más ancianos y mujeres ${ }^{19,20}$.

Educación e ingresos. A menor nivel educativo y de ingresos mayor posibilidad de presentar fragili$\operatorname{dad}^{19}$. Asimismo, la edad adulta (poca educación y ocupación) y las actuales condiciones sociales (ingresos insuficientes) se asocian a mayores probabilidades de fragilidad en ambos sexos ${ }^{17}$.

Estado civil. De acuerdo a Gobbens, ser mujer predice fragilidad social debido a su mayor probabilidad de vivir solas ${ }^{17}$. Asimismo, la viudez se asocia a fragilidad social ${ }^{20}$.

Estilos de vida. Existe evidencia sobre la influencia de un estilo de vida poco saludable sobre la fragilidad ${ }^{17}$. En relación a lo previo, se ha reportado que índices de masa corporal (IMC) mayores a $30^{15} \mathrm{y}$ adiposidad central fueron predictivos de fragilidad $^{21}$. Asimismo, un estudio realizado en Corea en el año 2014 estableció que los encuestados frágiles obesos mostraron mayor riesgo de 
mortalidad $^{22}$. Concordante con lo anterior, existe evidencia que establece que la multimorbilidad es un predictor de fragilidad ${ }^{17}$.

En cuanto a los tópicos etnicidad, ambiente y eventos vitales, no se encontró evidencia.

Enfermedad. Declinación de las reservas fisiológicas

Comorbilidad. Existe evidencia reciente en relación al rol de las enfermedades crónicas como predictoras de fragilidad ${ }^{19}$. Según un estudio realizado en Latinoamérica en el año 2008, se encontró que la comorbilidad se relacionaba con fragilidad ${ }^{15}$. Asimismo, en un estudio llevado a cabo en el año 2011 en Estados Unidos, se estableció que la fragilidad se asoció con depresión, accidentes cerebrovasculares, deterioro cognitivo, artritis y enfermedad pulmonar. Sumado a lo previo se concluyó que no existía relación con enfermedad cardíaca coronaria, diabetes mellitus y enfermedad renal ${ }^{15}$. En el modelo ajustado, la carga de enfermedad se mantuvo significativamente asociada con la fragilidad ${ }^{23}$. Sin embargo, en un estudio llevado a cabo en 2012 en Estados Unidos, se estableció que la diabetes mellitus con complicación macrovascular y menor educación son importantes predictores de la progresión en cualquier característica de fragilidad ${ }^{24}$.

En Japón, en el año 2012 se llevó a cabo un estudio que estableció que la presión del pulso, presencia de déficit cognitivo y auditivo se asociaban a mayor fragilidad ${ }^{25}$. Asimismo, en un estudio realizado en el año 2014 en Singapur, se asoció la edad mayor de 75 años, el no tener educación, insuficiencia cardíaca, trastornos respiratorios, accidente cerebrovascular, síntomas depresivos, deterioro auditivo, discapacidad visual, trastornos respiratorios, filtración glomerular inferior a 60 $\mathrm{ml} / \mathrm{min}$, puntuación de riesgo nutricional de 3 o superior, anemia, y recuentos de células blancas (> 6,5 o más) con fragilidad física ${ }^{26}$. Asimismo, de acuerdo a un estudio realizado en el año 2008 en Estados Unidos, se estableció que una función cognitiva inferior, anorexia y pérdida de peso eran predictores de fragilidad ${ }^{13}$.

De acuerdo un estudio realizado en Gran Bretaña en el año 2010, siete factores explican la asociación entre los indicadores de fragilidad. Entre ellos destacan la capacidad física, los síntomas o enfermedades cardiacas y respiratorias, las medidas fisiológicas, los problemas psicológicos, las comorbilidades y el deterioro visual ${ }^{27}$.
Asimismo, en Brasil en el año 2012, se identificó que la presencia de comorbilidad, dependencia en actividades básicas e instrumentales, así como en las actividades de la vida diaria eran factores asociados a fragilidad ${ }^{18}$.

Existe evidencia en Estados Unidos del año 2013 en la que se concluyó que pacientes con insuficiencia cardiaca y frágiles tenían un riesgo 2 veces mayor de muerte en comparación con aquellos que no tienen fragilidad ${ }^{28}$.

Percepción de salud. Una auto-percepción de salud negativa es predictor de fragilidad ${ }^{18,19}$.

\section{Fragilidad física, psicológica y social}

\section{Fragilidad física}

Existe consenso en relación a un estado de mayor vulnerabilidad asociado a factores de estrés secundario a la disminución de la reserva fisiológica relacionada con la edad (neuromuscular, metabólica y sistema inmune), sarcopenia y sistema nervioso ${ }^{29}$.

Declinación en nutrición, movilidad, actividad física, equilibrio, fuerza, resistencia y funciones sensoriales. La fragilidad se asoció con una mayor variabilidad de todos los parámetros de la marcha. La variabilidad del tiempo de andar rápido fue el parámetro más importante asociado consistentemente con fragilidad ${ }^{30}$. Asimismo, se encontró que esta se asociaba a bajo rendimiento en varios parámetros de marcha cuantitativa más allá de la velocidad, de los cuales el más destacado es la alta variabilidad del paso del tiempo ${ }^{30}$. Existe evidencia sobre la importancia de la movilidad, la edad y la fragilidad basal en las transiciones hacia fragilidad $^{31}$. Asimismo, se asocian a fragilidad la velocidad lenta de la marcha ${ }^{25-32}$.

Sumado a lo previo, en el año 2008 en un estudio realizado en Estados Unidos, se encontró que la velocidad de la marcha lenta, baja actividad física y pérdida de peso se asociaron independientemente a efectos adversos en salud como consecuencia de un estado de fragilidad ${ }^{33}$. Asimismo, de acuerdo a un estudio realizado en el año 2008 en el mismo país, el presentar marcha más lenta, una fuerza de agarre débil, y menor actividad física predijo mayor discapacidad y mortalidad ${ }^{13}$. Asimismo, existen parámetros tales como velocidad, impulso y orientación que son capaces de diferenciar entre adultos y poblaciones mayores con diferentes niveles de fragilidad ${ }^{34}$. 
La práctica de la actividad física, especialmente en el tiempo de ocio o acumulada en diferentes dominios, contribuye a la prevención de la fragilidad en las personas mayores ${ }^{35}$.

De acuerdo al índice de fragilidad (Frailty Index), las medidas de desempeño que se acercan más a la fragilidad pero diferente entre los distintos niveles de fragilidad eran la movilidad ambulatoria, la menor resistencia muscular del cuerpo y la fuerza de prensión no dominante ${ }^{36}$. Sin embargo, según un estudio realizado en el año 2011 en Canadá, los trastornos del equilibrio y movilidad contribuyen a fragilidad, pero tampoco es suficiente para definir a un participante como frágil ${ }^{37}$.

\section{Fragilidad psicológica}

Declinación en cognición, mecanismos de afrontamiento y estado de ánimo. Existe evidencia sobre la importancia de los eventos de la vida como predictores de fragilidad psicológica ${ }^{17}$. Sumado a lo previo, el deterioro cognitivo y la presencia de sintomatología depresiva predicen la condición de fragilidad ${ }^{38}$.

De acuerdo a evidencia del año 2008, el deterioro cognitivo también se asoció con discapacidad crónica, a uso de servicios de salud a largo plazo y la muerte, y la magnitud de estas asociaciones fue comparable con el de la pérdida de peso $^{33}$.

\section{Fragilidad social}

Declinación en relaciones sociales y apoyo social. El riesgo de fragilidad incidente entre los encuestados que participan en actividades físicas solitarias fue significativamente menor que en los que no participaron en estas actividades ${ }^{39}$.

\section{Innovaciones actuales en índices de predicción de fragilidad}

En el año 2010 fue llevado a cabo un estudio que buscaba proporcionar a los profesionales europeos de la comunidad un instrumento sencillo y válido que ofreciera un pre-cálculo de fragilidad según género de acuerdo a la introducción de los cinco criterios propuestos por Fried. Dicho instrumento denominado SHARE para fragilidad como constructo resultó ser suficiente y tener validez predictiva, ser fácil y de libre acceso a través de calculadoras $w e b^{40}$.

Morley desarrolló una escala en una comunidad de afroamericanos entre 49-65 años. Esta sencilla escala de 5 preguntas es una prueba de detección excelente dirigida a identificar a las personas frágiles en riesgo de desarrollar discapacidad, disminución en el funcionamiento y mortalidad $^{41}$. Llama la atención el grupo etario en el cual se validó.

En el año 2014, se llevó a cabo en Italia un estudio que tenía como objetivo desarrollar y probar un cuestionario de selección postal para identificar fragilidad en las personas mayores residentes en la comunidad. Se encontró que la capacidad de la puntuación del cuestionario para predecir la fragilidad era adecuada. Se concluyó que un sencillo cuestionario enviado por correo fue capaz de identificar fragilidad física en la comunidad. Esto facilitaría la detección de fragilidad a gran escala ${ }^{42}$.

La mayoría de los estudios encontrados fueron estudios cuantitativos de corte transversal y longitudinal. Se decidió incluir ambos tipos de estudios como una forma de hacer más amplia la búsqueda y pesquisar predictores comunes. Asimismo, gran parte de estos fueron realizados en Norteamérica y Europa. Lo anterior, puede ser considerado una limitante debido a que los resultados no necesariamente son extrapolables a la población chilena.

\section{Discusión}

Esta revisión de literatura permite describir los predictores asociados a la prevalencia de fragilidad en sus dominios físicos, psicológicos y sociales. A partir de la evidencia encontrada, es posible formular un perfil de las personas mayores frágiles (Tabla 1). La fragilidad fisca es una de las

Tabla 1. Resumen de perfil de fragilidad

\begin{tabular}{|ll|}
\hline Fragilidad & Criterios \\
Física & $\begin{array}{l}\text { Pérdida de peso involuntario, fuerza muscular, cansancio físico, percepción de estado de salud, equilibrio } \\
\text { y problema con las funciones de los órganos de los sentidos como visión y audición }\end{array}$ \\
\hline $\begin{array}{l}\text { Psicológica } \\
\text { Social }\end{array}$ & Cognición, síntomas depresivos, ansiedad y estrategias de enfrentamiento \\
\hline
\end{tabular}


más estudiadas y la que cuenta con mayor grado de evidencia científica a nivel mundial, siendo un eje principal de los distintos tipos de fragilidad. A pesar de que no existe una única forma de medición de la fragilidad física, hay consenso de los aspectos que influyen en ellas, como son: pérdida de peso involuntario, fuerza muscular, cansancio físico, percepción de estado de salud, equilibrio y problema con las funciones de los órganos de los sentidos como visión y audición ${ }^{17}$. Los esfuerzos a partir de esta revisión deben ser enfocados en el desarrollo de estudios que predigan fragilidad en sus dimensiones sociales y psicológicas con el fin de intervenir de manera integral en las personas mayores. Asimismo, se propone que se desarrollen intervenciones destinadas a la prevención de esta.

Según Gobbens, la fragilidad psicológica ha sido definida por: cognición, síntomas depresivos, ansiedad y estrategias de enfrentamiento ${ }^{17}$. El deterioro cognitivo ha sido respaldado como un indicador esencial de la fragilidad en los $\mathrm{AM}^{33}$. Asimismo, ha sido descrito ampliamente en la literatura científica ${ }^{29,43}$.

En relación a la fragilidad social, ha sido menos estudiada y es un área abierta para futuras investigaciones. Los factores sociales afectan los indicadores de salud de los AM, describiéndose la idea subyacente de que la reserva de déficit o problemas que acumula una persona a lo largo de la vida, lo hace más propenso a presentar problemas de salud ${ }^{44,45}$. Los determinantes de la fragilidad social se han definido como: vivir solo, relaciones y apoyo social ${ }^{17}$.

Finalmente, tanto la fragilidad física como psicológica y social tienen una compleja interrelación que afectan los resultados de salud. Se ha demostrado que la fragilidad física y la fragilidad psicológica (deterioro cognitivo) están asociadas, en estudios longitudinales que han mostrado que la fragilidad física está asociado a un deterioro cognitivo a los 4 años de seguimiento ${ }^{46}$, siendo la fragilidad física un factor de riesgo de deterioro cognitivo en los $\mathrm{AM}^{47,48}$.

Finalmente, futuras investigaciones debieran centrarse en tres aspectos importantes. El primero de ellos es avanzar en la descripción de la fragilidad psicológica y social con más investigaciones del área social, de forma de definir un perfil claro en esos aspectos de la fragilidad. Segundo, es un desafío importante centrar la atención en la detección de mujeres adultas mayores con patologías asociadas, de bajos ingresos, con un IMC aumentado, sedentarias, con alteraciones de la marcha, viudas, con trastornos como depresión o deterioro cognitivo y con autopercepción de salud negativa. $Y$, finalmente, es necesario avanzar en instrumentos de medición que incluyan a la fragilidad en sus tres dimensiones y que sean un Gold estándar para definir las futuras investigaciones en esta área temática.

\section{Referencias}

1. Gobbens R, van Assen M, Luijkx K, Schols J. The predictive validity of the Tilburg Frailty Indicator: Disability, health care utilization, and quality of life in a population at risk. Gerontologist 2012; 52 (5): 619-31.

2. Gobbens R, Luijkx K, Wijnen-Sponselee M, Schols J. Toward a conceptual definition of frail community dwelling older people. Nurs Outlook 2010; 58: 76-86.

3. Ensrud KE, Ewing SK, Cawthon PM, Fink HA, Taylor BC, Cauley JA, et al. A comparison of frailty indexes for the prediction of falls, disability, fractures, and mortality in older men. J Am Geriatr Soc 2009; 57 (3): 492-8.

4. Song X, Mitnitski A, Rockwood K. Prevalence and 10 -year outcomes of frailty in older adults in relation to deficit accumulation. J Am Geriatr Soc 2010; 58 (4): 681-7.

5. Alvarado BE, Zunzunegui MV, Beland F, Bamvita JM. Life course social and health conditions linked to frailty in Latin American older men and women. J Gerontol A Biol Sci Med Sci. [Multicenter Study].

6. Semba RD, Barthali B, Zhou J, Blaum C, Chia-Wen K, Fried L. Low Serum Micronutrient Concentrations Predict Frailty Among Older Women Living in the Community. J Geriatr 2006; 61A (6): 594-9.

7. Qu T, Yang H, Walston J, Fedarko N, Leng S. Upregulated monocytic expression of CXC chemokine ligand 10 (CXCL-10) and its relationship with serum interleukin-6 levels in the syndrome of frailty. Cytokine 2009; 46: 319-24.

8. Le Couteur D, Blyth F, Creasey H, Handelsman D, Naganathan V, Sambrook P, et al. The Association of Alanine Transaminase With Aging, Frailty, and Mortality. J Gerontol A Biol Sci Med Sci 2010; 65 (7): 712-7.

9. Travison T, Nguyen A, Naganathan V, Stanaway F, Blyth F, Cumming R, et al. Changes in Reproductive Hormone Concentrations Predict the Prevalence and Progression of the Frailty Syndrome in Older Men: 
The Concord Health and Ageing in Men Project. J Clin Endocrinol Metab 2011; 96 (8): 2464-74.

10. Baylis D, Barlett BD, Syddall HE, Ntani G, Gale CR, Cooper $\mathrm{C}$, et al. Immune-endocrine biomarkers as predictors of frailty and mortality: a 10-year longitudinal study in community-dwelling older people. J Am Aging Assoc 2013; 35: 963-71.

11. Voznesensky M, Walsh S, Dauser D, Brindisi J, Kenny AM. The association between dehydroepiandosterone and frailty in older men and women. Age Ageing 2009; 38: 401-6.

12. Leng S, Xue Q, Tian J, Huang Y, Yeh S, Fried L. Associations of neutrophil and monocyte counts with frailty incommunity-dwelling disabled older women: Results from the Women's Health and Aging Studies I. Exp Gerontol 2009; 44: 511-6.

13. Sarkisian CA, Gruenewald T, Boscardin WJ, Seeman TE. Preliminary Evidence for Subdimensions of Geriatric Frailty: The MacArthur Study of Successful Aging. J Am Geriatr Soc 2008; 56: 2292-7.

14. Dalrymple LS, Katz R, Rifkin DE, Siscovick D, Newman AB, Fried LF, et al. Kidney Function and Prevalent and Incident Frailty. Clin J Am Soc Nephrol 2013; 8: 2091-9.

15. Alvarado B, Zunzunegui M, Beland F, Bamvita JM. Life Course Social and Health Conditions Linked to Frailty in Latin American Older Men and Women. Journal of Gerontology 2008; 63 (12): 1399-406.

16. Song X, Mitnitski A, Rockwood K. Prevalence and 10Year Outcomes of Frailty in Older Adults in Relation to Deficit Accumulation. J Am Geriatr Soc 2010; 58: 681-7.

17. Gobbens RJ, van Assen MA, Luijkx KG, Wijnen-Sponselee MT, Schols JM. Determinants of frailty. J Am Med Dir Assoc 2010; 11 (5): 356-64.

18. Sousa AC, Dias R, Maciel ÁC, Guerra RO. Frailty syndrome and associated factors in community-dwelling elderly in Northeast Brazil. Arch Gerontol Geriatr 2012; 54: e95-e101.

19. Aguilar-Navarro SG, Amieba H, Gutiérrez-Robledo LM, Ávila-Funes JA. Frailty among Mexican community-dwelling elderly: a story told 11 years later. The Mexican Health and Aging Study. Salud Publica Mex 2015; 57: S62-S9.

20. Garre-Olmo J, Calvó-Perxas L, López-Pousa S, de Gracia Blanco M, Vilalta-Franch J. Prevalence of frailty phenotypes and risk of mortality in a community-dwelling elderly cohort. Age Ageing 2013; 42: 46-51.

21. Sheehan KJ, O’Connell MD, Kenny RA. 100 central adiposity is an independent predictor for the development of frailty in community dwelling older adults. Age Ageing 2014; 43: i27.

22. Lee Y, Kim J, Han ES, Ryu M, Cho Y, Chae S. Frailty and body mass index as predictors of 3-year mortality in older adults living in the community. Gerontology. 2014; 60 (6): 475-82.

23. Sanders J, Boudreau R, Fried L, Walston J, Harris T, Newman A. Measurement of Organ Structure and Function Enhances Understanding of the Physiological Basis of Frailty: The Cardiovascular Health Study. J Am Geriatr Soc 2011; 59: 1581-8.

24. Espinoza SE, Jung I, Hazuda H. Frailty Transitions in the San Antonio Longitudinal Study of Aging. J Am Geriatr Soc 2012; 60: 652-60.

25. Doba N, Tokuda N, Goldstein N, Kushiro T, Hinohara S. A pilot trial to predict frailty syndrome: The Japanese Health Research Volunteer Study. Exp Gerontol 2012; 47: 638-43.

26. Ng TP, Feng L, Nyunt MS, Larbi A, Yap KB. Frailty in Older Persons: Multisystem Risk Factors and the Frailty Risk Index (FRI). JAMDA 2014; 15: 635-42.

27. Kamaruzzaman S, Ploubidis G, Fletcher A, Ebrahim S. A reliable measure of frailty for a community dwelling older population. Health Qual Life Outcomes 2010; 8: 123.

28. McNallan SM, Chamberlain AM, Gerber Y, Singh M, Kane RL, Weston SA, et al. Measuring frailty in heart failure: A community perspective. Am Heart J 2013; 166 (4): 768-74.

29. Kuh D. A life course approach to healthy aging, frailty, and capability. J Gerontol A Biol Sci Med Sci [Editorial Review]. 2007; 62 (7): 717-21.

30. Montero-Odasso M, Muir S, Hall M, Doherty T, Kloseck M, Beauchet O, et al. Gait Variability Is Associated With Frailty in Community-dwelling Older Adults. J Gerontol A Biol Sci Med Sci 2011; 66a (5): 568-76.

31. Fallah N, Mitnitski A, Searle SD, Gahbauer E, Gill T, Rockwood K. Transitions in Frailty Status in Older Adults in Relation to Mobility: A Multi-State Modeling Approach Employing a Deficit Count. J Am Geriatr Soc 2011; 59 (3): 524-9.

32. Verghese J, Holtzer R, Lipton RB, Wang C. Mobility stress test approach to predicting frailty, disability, and mortality in high-functioning older adults. J Am Geriatr Soc 2012; 60 (10): 1901-5.

33. Rothman MD, Leo-Summers L, Gill TG. Prognostic Significance of Potential Frailty Criteria. J Am Geriatr Soc 2008; 56: 2211-6.

34. Millor N, Lecumberri P, Gómez M, Martínez-Ramírez A, Izquierdo M. An evaluation of the 30-s chair stand test in older adults: frailty detection based on kinematic parameters from a single inertial unit. J Neuroeng Rehabil 2013; 10: 86. 
35. Tribess S, Virtuoso Junior JS, Oliveira RJ. Physical activity as a predictor of absence of frailty in the elderly. Rev Assoc Med Bras 2012; 58 (3): 341-7.

36. Theou O, Jones G, Jakobi J, Mitnitski A, Vandervoort A. A comparison of the relationship of 14 performance-based measures with frailty in older women. Appl Physiol Nutr Metab 2011; 36: 928-38.

37. Davis DH, Rockwood MR, Mitnitskia A, Rockwood $\mathrm{K}$. Impairments in mobility and balance in relation to frailty. Arch Gerontol Geriatr 2011; 53: 79-83.

38. Duarte M, Paul C. Indicadores de saúde mental como fatores preditores de fragilidade nos idiosos. Revista Portuguesa de Enfermagem de Saúde Mental 2014; 1 : 27-32.

39. Fushiki Y, Ohnishi H, Sakauchi F, Oura A, Mori M. Relationship of Hobby Activities With Mortality and Frailty Among Community-Dwelling Elderly Adults: Results of a Follow-up Study in Japan. J Epidemiol 2012; 22 (4): 340-7.

40. Romero-Ortuno R, Walsh CD, Lawlor B, Kenn RA. A Frailty Instrument for primary care: findings from the Survey of Health, Ageing and Retirement in Europe (SHARE). BMC Geriatrics 2010; 10: 57.

41. Morley JE, Malmstrom T, Miller DK. A simple frailty questionnaire (FRAIL) predicts outcomes in middle aged African Americans. Nutr Health Aging 2012; 16 (7): 601-8.
42. Di Bari M, Profili F, Bandinelli S, Salvioni A, Mossello E, Corridori C, et al. Screening for Frailty in Older Adults Using a Postal Questionnaire: Rationale, Methods, and Instruments Validation of the INTER-FRAIL Study. J Am Geriatr Soc 2014; 62: 1933-7.

43. Fried LP, Ferrucci L, Darer J, Williamson JD, Anderson G. Untangling the concepts of disability, frailty, and comorbidity: implications for improved targeting and care. J Gerontol A Biol Sci Med Sci 2004; 59 (3): 25563.

44. Andrew MK, Rockwood K. Social vulnerability predicts cognitive decline in a prospective cohort of older Canadians. Alzheimer's \& Dementia 2010; 6 (4): 319-25.

45. Andrew MK, Mitnitski AB, Rockwood K. Social vulnerability, frailty and mortality in elderly people. PLoS One 2008; 3 (5): e2232.

46. Auyeung TW, Lee J, Kwok T, Woo J. Physical frailty Predicts future cognitive decline-a four-year ProsPective study in 2737 cognitively normal older adults. J Nutr Health Aging 2011; 15 (8): 690-4.

47. Mitnitski A, Fallah N, Rockwood MR, Rockwood K. Transitions in cognitive status in relation to frailty in older adults: a comparison of three frailty measures. J Nutr Health Aging 2011; 15 (10): 863-7.

48. Jacobs JM, Cohen A, Ein-Mor E, Maaravi Y, Stessman J. Frailty, cognitive impairment and mortality among the oldest old. J Nutr Health Aging 2011; 15 (8): 678-82. 